DOI: $10.17516 / 1999-494 X-0250$

УДК 621.314:621.43:536.2

\title{
The Mathematical Model and Numerical Study \\ of Heat and Mass Transfer Processes \\ in the Combustion Chamber of Diesel-Generator Units \\ with a Valve-Inductor Generator
}

\author{
Dmitriy V. Guzei*a, Andrey V. Minakova,b, \\ Vasiliy I. Panteleev ${ }^{a}$, Maksim I. Pryazhnikova,b, \\ Dmitriy V. Platonov ${ }^{\mathrm{a}, \mathrm{b}}$ and Vladimir A. Zhigarev ${ }^{\mathrm{a}}$ \\ ${ }^{a}$ Siberian Federal University \\ Krasnoyarsk, Russian Federation \\ ${ }^{b}$ Kutateladze Institute of Thermophysics $S B R A S$ \\ Novosibirsk, Russian Federation
}

Received 14.02.2020, received in revised form 19.04.2020, accepted 21.05.2020

Abstract. The mathematical model of heat and mass transfer processes in the combustion chamber of diesel generator units with valve inductor generators has been developed. The mathematical model takes into account the actual geometry of the combustion chamber and the operating conditions of the diesel engine. A study of the main characteristics of a diesel generator in a wide range of modes of operation has been carried out. In addition to energy characteristics, environmental parameters have been considered.

Keywords: diesel engine, a valve-inductor generator, variable speed, a combustion chamber, emissions, optimization, a mathematical model.

Citation: Guzei D.V., Minakov A.V., Panteleev V.I., Pryazhnikov M.I., Platonov D.V., Zhigarev V.A. The mathematical model and numerical study of heat and mass transfer processes in the combustion chamber of diesel-generator units with a valveinductor generator, J. Sib. Fed. Univ. Eng. \& Technol., 2020, 13(5), 611-625. DOI: 10.17516/1999-494X-0250

(C) Siberian Federal University. All rights reserved

This work is licensed under a Creative Commons Attribution-Non Commercial 4.0 International License (CC BY-NC 4.0).

* Corresponding author E-mail address: DGuzey@sfu-kras.ru 


\title{
Математическая модель и расчетное исследование процессов тепломассообмена в камере сгорания
}

\author{
дизель-генераторных установок
}

\section{с вентильно-индукторным генератором}

\author{
Д.В. Гузейа ${ }^{\mathrm{a}}$, А.В. Минаков ${ }^{\mathrm{a}, \boldsymbol{\sigma}}$, В.И. Пантелеев ${ }^{\mathrm{a}}$, \\ М.И. Пряжников ${ }^{\mathrm{a}, \tilde{\sigma}}$, Д.В. Платонов ${ }^{\mathrm{a}, \tilde{\sigma}}$, В.А. Жигарев ${ }^{\mathrm{a}}$ \\ ${ }^{a}$ Сибирский федеральный университет \\ Российская Федерачия, Красноярск \\ ${ }^{6}$ Институт теплофизики им. С.С. Кутателадзе СО РАН \\ Российская Федерачия, Новосибирск
}

\begin{abstract}
Аннотация. Разработана математическая модель процессов тепломассообмена в камере сгорания дизель-генераторных установок с вентильно-индукторными генераторами. Математическая модель учитывает реальную геометрию камеры сгорания и условия работы дизельного двигателя. Проведено исследование основных характеристик дизель-генератора в широком диапазоне режимов его работы. При этом рассмотрены не только его энергетические характеристики, но и экологические параметры.

Ключевые слова: дизельный двигатель, вентильно-индукторный генератор, переменная частота вращения, камера сгорания, выбросы, оптимизация, математическая модель.

Цитирование: Гузей, Д.В. Математическая модель и расчетное исследование процессов тепломассообмена в камере сгорания дизель-генераторных установок с вентильно-индукторным генератором / Д.В. Гузей, А.В. Минаков, В.И. Пантелеев, М.И. Пряжников, Д.В. Платонов, В.А. Жигарев // Журн. Сиб. федер. ун-та. Техника и технологии, 2020. 13(5). С. 611-625. DOI: 10.17516/1999-494X-0250
\end{abstract}

\section{Введение}

Значительная часть территории Красноярского края представляет собой удаленные энергетически изолированные районы, в которых для электроснабжения используются дизель-генераторные установки (ДГУ), работающие на дизельном топливе, что требует значительных (около 3 млрд руб.) ежегодных дотаций из бюджета края для реализации доставки дизельного топлива и выравнивания тарифов на электроэнергию для ее потребителей. Всего на территории края работают 118 дизельных электростанций, имеющих в своем составе более 400 ДГУ. Сами установки электроснабжения, в силу суровых условий северных широт, должны обладать повышенной надежностью, ремонтопригодностью, экономичностью и универсальностью. Дизель-генераторные установки традиционно используются в качестве локальных источников генерации электроэнергии. При этом одним из основных требований к ним является поддержание стабильной частоты вращения при изменении электрической нагрузки в достаточно широком диапазоне, что обусловлено необходимостью поддержания стабильных значений частоты и напряжения генерируемой электроэнергии и обеспечивается соответствующим построением регуляторов частоты вращения вала приводного двигателя.

Чаще всего такие источники генерации большую часть времени работают на нагрузку меньше номинальной, что приводит к завышенному потреблению дизельного топлива и к со- 
кращению ресурса работы дизельного двигателя. Кроме того, подавляющее большинство дизель-генераторов работает со стабильной скоростью вращения вала дизеля независимо от мощности нагрузки. Такой режим работы дизеля также не оптимален с точки зрения потребления топлива.

В ряде работ для экономии дизельного топлива и продления ресурса двигателя предлагается регулировать его частоту вращения вала в функции нагрузки электрической сети [1-5]. Однако при этом остается необходимость обеспечения стабильных значений напряжения и частоты в электрической сети, питающей потребителей электроэнергии. Этим и другим требованиям в большей степени соответствуют разработанные в последнее время дизель-генераторные установки с переменной частотой вращения. Это новый тип генерирующего оборудования с использованием вентильно-индукторных генераторов, имеющего ряд преимуществ перед широко применяемыми сегодня ДГУ с синхронными генераторами, основные из которых по-

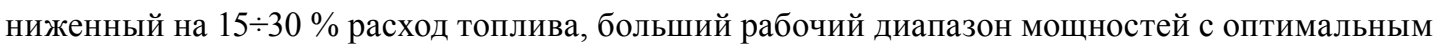
режимом привода, больший ресурс оборудования.

Исследования, проведенные в семидесятые-восьмидесятые годы прошлого века под руководством А.В. Орлова [1], В.И. Крутова [2] и развитые в более поздних работах [3-5], показали, что уменьшение частоты вращения ДВС при снижении мощности нагрузки позволяет сократить удельный расход топлива на 20-30 \%. Вариация частоты вращения вала при изменении мощности нагрузки ДВС также обеспечивает оптимальный тепловой режим, снижает износ и, следовательно, повышает моторесурс.

Следует заметить, что дизельные двигатели с момента их применения всегда оснащались регуляторами частоты вращения коленчатого вала, но поскольку они изначально предназначались для оснащения транспортных средств различного назначения (автомобили, тракторы, машины военного применения и т.п.), то главной задачей таких регуляторов было и остается обеспечение стабильной заданной частоты вращения при изменяющейся нагрузке, а изменение задания частоты вращения при этом предполагается вручную посредством специального устройства. В дизельных двигателях дизель-генераторных установок с синхронными генераторами задача регулятора принципиально сохраняется прежней с той лишь разницей, что частота вращения всегда должна оставаться постоянной, чтобы обеспечивать неизменной частоту генерируемого напряжения.

При автоматическом регулировании частоты вращения дизельного двигателя в камере сгорания происходят переходные процессы. Двигатель работает на частоте вращения, отличной от номинальной, вследствие этого могут возрастать выбросы, повышаться расход топлива. Также необходимо учитывать изменение тепловыделения двигателя на различных режимах работы и нагрузки. Для оптимизации работы дизельных двигателей в составе дизель-генераторных установок с переменной частотой вращения используется математическое моделирование.

В настоящее время известны несколько упрощенных математических моделей, описывающих параметры работы дизель-генераторов с переменной частотой вращения. Здесь особо следует отметить работы, выполненные под руководством О.С. Хватова $[6,7]$. Данные модели основаны на решении системы обыкновенных дифференциальных уравнений для двигателя и генератора с эмпирическими набором параметров и описывают поведение интегральных ха- 
рактеристик системы. Локальные характеристики работы двигателя и генератора эти модели не представляют. Однако проектирование современных энергетических установок требует описания локальных параметров системы. Поэтому необходима разработка комплексной математической модели, которая могла бы описывать как интегральные, так и локальные параметры установки.

Целью настоящего исследования является разработка математической модели процессов тепломассообмена в камере сгорания дизель-генераторных установок с вентильно-индукторными генераторами. Данная работа является первым шагом к созданию полноценного цифрового двойника дизель-генераторной установки с переменной частотой вращения.

\section{1. Математическая модель}

Для моделирования процессов, происходящих в камере сгорания двигателя дизель-генератора с переменной частотой вращения, была разработана математическая модель процессов горения в камере сгорания с учетом характеристик впрыска, воспламенения и сгорания топливной смеси. Модель была разработана на основе пакета программ CFD ANSYS® Forte.

Математическая модель процессов тепломассобмена в камере сгорания двигателя состоит из следующих уравнений.

Уравнение сохранения массы газовой смеси:

$$
\frac{\partial \bar{\rho}}{\partial t}+\nabla \cdot(\bar{\rho} \tilde{\boldsymbol{u}})=\dot{\bar{\rho}}^{s}
$$

где $\widetilde{\boldsymbol{u}}$ - вектор скорости потока; $\bar{\rho}$ - плотность смеси; $\bar{\rho}_{k}^{s}-$ источник массы в процессе испарения дизельного топлива.

Уравнение сохранения импульса смеси учитывает свободную конвекцию, силы давления, силы вязкого трения, турбулентные напряжения, а также импульс, вносимый при распыливании спрея дизельного топлива в камере сгорания:

$$
\frac{\partial \bar{\rho} \tilde{u}}{\partial t}+\nabla \cdot(\bar{\rho} \widetilde{u} \tilde{u})=-\nabla \bar{p}+\nabla \cdot \overline{\boldsymbol{\sigma}}-\nabla \cdot \Gamma+\overline{\mathbf{F}}^{s}+\bar{\rho} \overline{\mathbf{g}}
$$

где $\bar{p}$ - давление; $\bar{F}^{s}$ - сила, вносимая в единицу объема при распылении топлива; $g$ - ускорение свободного падения; $\Gamma=\bar{\rho}(\widetilde{\boldsymbol{u}} \tilde{\boldsymbol{u}}-\tilde{\boldsymbol{u}} \tilde{u})-$ тензор рейнольдсовых напряжений, который в рамках RANS подхода определяется согласно гипотезе Буссинеска; $\bar{\sigma}$ - тензор вязких напряжений, определяемый как

$$
\overline{\boldsymbol{\sigma}}=\bar{\rho} v\left[\nabla \tilde{\boldsymbol{u}}+(\nabla \tilde{\boldsymbol{u}})^{\mathrm{T}}-\frac{2}{3}(\nabla \cdot \tilde{\boldsymbol{u}}) \mathbf{I}\right],
$$

где $v$ - кинематическая вязкость среды; I - единичный тензор.

Для моделирования турбулентности использована нестационарная $\mathrm{k}-\varepsilon$ RANS-модель для нормализованных групп (RNG) с дополнительными членами $\dot{\bar{W}}^{s}$, учитывающими взаимодействие турбулентных вихрей и капель спрея [8].

$$
\frac{\partial \bar{\rho} \tilde{k}}{\partial t}+\nabla \cdot(\bar{\rho} \tilde{u} \tilde{k})=-\frac{2}{3} \bar{\rho} \tilde{k} \nabla \cdot \tilde{u}+(\bar{\sigma}-\Gamma): \nabla \tilde{u}+\nabla \cdot\left\lceil\frac{\left(\mu+\mu_{T}\right)}{P r_{k}} \nabla \tilde{k}\right\rceil-\bar{\rho} \tilde{\varepsilon}+\dot{\bar{W}}^{s}
$$




$$
\begin{aligned}
\frac{\partial \bar{\rho} \tilde{\varepsilon}}{\partial t}+\nabla \cdot(\bar{\rho} \tilde{u} \tilde{\varepsilon}) & = \\
= & -\left(\frac{2}{3} c_{\varepsilon 1}-c_{\varepsilon 3}\right) \bar{\rho} \tilde{\varepsilon} \nabla \cdot \tilde{u}+\nabla \cdot\left[\frac{\left(v+v_{T}\right)}{P r_{\varepsilon}} \nabla \tilde{\varepsilon}\right]+ \\
+ & \frac{\tilde{\varepsilon}}{\tilde{k}}\left(c_{\varepsilon 1}(\bar{\sigma}-\Gamma): \nabla \tilde{u}-c_{\varepsilon 2} \bar{\rho} \tilde{\varepsilon}+c_{s} \dot{\bar{W}}^{s}\right), \tilde{k}
\end{aligned}
$$

где $\tilde{k}, \tilde{\varepsilon}$ - кинетическая энергия турбулентных пульсаций и скорость диссипации турбулентных пульсаций.

Уравнение сохранения энергии учитывает работу сил давления, конвекцию и теплопроводность, турбулентную диссипацию энергии, тепловыделение в процессе химических реакций и испарения топлива и записывается для внутренней энергии в следующем виде:

$$
\frac{\partial \bar{\rho} \tilde{\mathbf{I}}}{\partial t}+\nabla \cdot(\bar{\rho} \tilde{\boldsymbol{u}} \tilde{\mathbf{I}})=-\bar{p} \nabla \cdot \tilde{\boldsymbol{u}}-\nabla \cdot \overline{\mathbf{J}}-\nabla \cdot \mathbf{H}+\bar{\rho} \tilde{\varepsilon}+\dot{\bar{Q}}^{C}+\dot{\bar{Q}}^{s},
$$

где I - удельная внутренняя энергия; $\mathbf{\mathbf { J }}$ - вектор теплового потока, который определяется как

$$
\overline{\mathbf{J}}=-\lambda \nabla \bar{T}-\bar{\rho} D \sum_{k} \widetilde{\boldsymbol{h}}_{k} \nabla \bar{y}_{k},
$$

где $\lambda$ - теплопроводность смеси; $T$ - температура; $\bar{y}_{k}$ - массовая доля компонент смеси; $h_{k}-$ удельная энтальпия; $\dot{\bar{Q}}^{c}$ и $\dot{\bar{Q}}^{S}$ - источниковые члены, связанные с тепловыделением в процессе горения и испарения топлива соответственно; $H$ - турбулентный поток тепла.

Уравнение сохранения массы для химического вещества $k$ имеет вид

$$
\frac{\partial \bar{\rho} \bar{y}_{k}}{\partial t}+\nabla \cdot\left(\bar{\rho} \bar{y}_{k} \tilde{\boldsymbol{u}}\right)=\nabla \cdot\left[\bar{\rho} D \nabla \bar{y}_{k}\right]+\nabla \cdot \Phi+\dot{\bar{\rho}}_{k}^{c}+\dot{\bar{\rho}}_{k}^{s} \quad(k=1, \cdots, K) .
$$

Здесь $K$ - общее число компонентов смеси; $D$ - эффективный коэффициент диффузии с учетом турбулентного переноса; $\dot{\bar{\rho}}_{\mathrm{k}}^{\mathrm{c}}$ и $\dot{\bar{\rho}}_{\mathrm{k}}^{\mathrm{s}}-$ источники массы $\kappa$-го компонента смеси в процессе химических реакций и испарения из топлива соответственно.

Для моделирования распыливания дизельного топлива использовали модель полного конуса, которая включает в себя распыливание топлива, дробление его капель, их столкновение и слияние, испарение. При этом начальные характеристики спрея (диаметр капель и их скорость) определяли эмпирически с помощью коэффициента расхода форсунки. Для моделирования дальнейшего распада и дробления капель топлива использована модель Рэлея-Тейлора [9].

Для моделирования испарения капель дизельного топлива применили многокомпонентную модель. Данная модель испарения рассматривает сферическую каплю жидкости, которая состоит из конечного числа компонентов, испаряющихся без химических реакций в газовой среде [10].

Скорость образования массы $к$-го компонента смеси в процессе химических реакций определяли суммированием по всем реакциям, в которых участвует этот компонент:

$$
\dot{\bar{\rho}}_{k}^{c}=W_{k} \sum_{i=1}^{l} \dot{\omega}_{k i},
$$


где $\omega_{k i}$ - скорость образования к-го компонента смеси в і-й реакции; $W_{k}-$ молекулярная масса. При этом дизельное топливо представлялось моделью суррогатного топлива $-\mathrm{n}$-гептан $\left(\mathrm{nC}_{7}\right.$ $\mathrm{H}_{16}$ ). Для моделирования кинетики горения дизельного топлива в газовой фазе использовали кинетический механизм (Diesel_1COMp_35sp_CHeM.iNp), содержащий 74 элементарные реакции и 35 газовых компонент. Аррениусовские параметры $K=A T^{b} \exp (-E / R T)$ скорости рассматриваемых реакций приведены в табл. 1.

Для моделирования влияния турбулентности на кинетику горения топлива использовали обобщенную модель взаимодействия турбулентности и химии из работы Kong et al. [11], которая широко применяется для моделирования дизельных двигателей. Эта модель предполагает, что скорость химической реакции частично ограничивается скоростью турбулентного перемешивания. Для этого в модели вводится эффективный масштаб времени $\tau_{\text {eff; }}$ который складывается из характерного масштаба времени перемешивания $\tau_{m i x}$ и характерного масштаба времени химического реагирования $\tau_{c h e m}$. Соотношение между эффективной скоростью образования $\kappa$-го компонента $\widetilde{\tilde{\omega}}_{k, e f f}$ и скоростью его образования согласно кинетического механизма $\widetilde{\tilde{\omega}}_{k}$ определяется в этой модели как

$$
\tilde{\tilde{\omega}}_{k, \text { eff }}=\left(y_{k}^{n+1}-y_{k}^{n}\right) / \Delta t=\frac{\tau_{\text {chem }} \widetilde{\omega}_{k} \Delta t}{\tau_{\text {eff }}}=\frac{\tau_{\text {chem }}\left(y_{k}^{\kappa i n}-y_{k}^{n}\right)}{\left(\tau_{\text {chem }}+\tau_{m i x}\right) \Delta t},
$$

здесь $\Delta t-$ величина временного шага; $y_{k}^{n}$ - массовая концентрация на предыдущем временном шаге.

Для моделирования образования сажи в камере сгорания дизельного двигателя применили двухстадийную модель, состоящую из конкурирующих механизмов образования [12] и окисления [13] частиц сажи. Основные уравнения для модели сажи следующие:

$$
\begin{aligned}
& \frac{d M_{\mathrm{s}}}{d t}=\frac{d M_{\mathrm{sf}}}{d t}-\frac{d M_{\mathrm{so}}}{d t}, \\
& \frac{M_{\mathrm{sf}}}{d t}=K_{f} M_{\text {pre }}, \\
& K_{f}=A_{\mathrm{sf}} p^{n} \exp \left(-E_{f} / R T\right), \\
& \frac{d M_{\mathrm{so}}}{d t}=\frac{6 M W_{c}}{\rho_{s} D_{s}} M_{s} R_{\text {total }},
\end{aligned}
$$

где $p$ - давление смеси; $n=0,5$ - эмпирическая константа; $M_{\mathrm{s}}$ - равновесная масса сажи; $M_{\mathrm{sf}}-$ $M_{\mathrm{so}}$ - масса образовавшейся и окислившейся сажи; $M_{\mathrm{pre}}-$ масса прекурсора сажи; $K_{f}-$ скорость образования сажи; $A_{\mathrm{sf}}=40 \mathrm{~cm}^{3} /$ моль* образования сажи; $E_{f}=12500$ кал/моль - энергия активации образования сажи; $M W_{c}$ - моле-

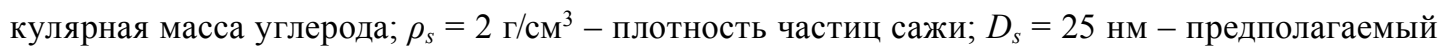
диаметр частиц сажи; $R_{\text {total }}$ - скорость окисления.

Дискретизация уравнений переноса осуществлялась по методу контрольного объема на неструктурированной сетке. Связь полей скорости и давления для несжимаемой жидкости реализовывалась с помощью процедуры SIMPLE. Для аппроксимации конвективных членов уравнения на компоненты импульса применена противопоточная схема второго порядка QSOU. Для аппроксимации конвективных членов уравнения на турбулентные характеристики использована противопоточная схема второго порядка. Нестационарные слагаемые аппрокси- 
Таблица 1. Параметры кинетического механизма горения модельного топлива

Table 1. Parameters of the combustion kinetic mechanism of model fuel

\begin{tabular}{|c|c|c|c|c|c|c|c|c|c|}
\hline № & Реакция & $\begin{array}{l}A, \text { mol- } \\
\mathrm{cm}-\mathrm{s}-\mathrm{K}\end{array}$ & $b$ & $\begin{array}{c}E, \\
\mathrm{cal} / \mathrm{mol}\end{array}$ & № & Реакция & $\begin{array}{l}A, \text { mol- } \\
\mathrm{cm}-\mathrm{s}-\mathrm{K}\end{array}$ & $b$ & $\begin{array}{c}E, \\
\mathrm{cal} / \mathrm{mol}\end{array}$ \\
\hline 1 & $\mathrm{nC}_{7} \mathrm{H}_{16}+\mathrm{H}=\mathrm{C}_{7} \mathrm{H}_{15}-2+\mathrm{H}_{2}$ & $4.38 \cdot 10^{7}$ & 2.0 & 4760.0 & 38 & $\mathrm{CH}_{3}+\mathrm{CH}_{3} \mathrm{O}=\mathrm{CH}_{4}+\mathrm{CH}_{2} \mathrm{O}$ & $4.30 \cdot 10^{14}$ & 0.0 & 0.0 \\
\hline 2 & $\mathrm{nC}_{7} \mathrm{H}_{16}+\mathrm{OH}=\mathrm{C}_{7} \mathrm{H}_{15}-2+\mathrm{H}_{2} \mathrm{O}$ & \begin{tabular}{|c|}
070.109 \\
\end{tabular} & 1.3 & 90.0 & 9 & $\mathrm{C}_{2} \mathrm{H}_{4}+\mathrm{OH}=\mathrm{CH}_{2} \mathrm{O}+\mathrm{CH}_{3}$ & $6.00 \cdot 10^{13}$ & 0.0 & 60.0 \\
\hline 3 & $\mathrm{nC}_{7} \mathrm{H}_{16}+\mathrm{HO}_{2}=\mathrm{C}_{7} \mathrm{H}_{15}-2+\mathrm{H}_{2} \mathrm{O}_{2}$ & $1.65 \cdot 10^{13}$ & 0.0 & 16950.0 & 40 & $\mathrm{C}_{2} \mathrm{H}_{4}+\mathrm{OH}=\mathrm{C}_{2} \mathrm{H}_{3}+\mathrm{H}_{2} \mathrm{O}$ & $8.02 \cdot 10^{13}$ & 0.0 & 5955.0 \\
\hline 4 & $\mathrm{nC}_{7} \mathrm{H}_{16}+\mathrm{O}_{2}=\mathrm{C}_{7} \mathrm{H}_{15}-2+\mathrm{HO}_{2}$ & $2.00 \cdot 10^{15}$ & 0.0 & 7380.0 & 41 & $\mathrm{C}_{2} \mathrm{H}_{3}+\mathrm{O}_{2}=\mathrm{CH}_{2} \mathrm{O}+\mathrm{HCO}$ & $4.00 \cdot 10^{12}$ & 0.0 & -250.0 \\
\hline 5 & $\mathrm{C}_{7} \mathrm{H}_{15}-2+\mathrm{O}_{2}=\mathrm{C}_{7} \mathrm{H}_{15} \mathrm{O}_{2}$ & 12 & 0.0 & 0 & 42 & $\mathrm{C}_{2} \mathrm{H}_{3}+\mathrm{HCO}=\mathrm{C}_{2} \mathrm{H}_{4}+\mathrm{CO}$ & $10^{13}$ & 0.0 & .0 \\
\hline 6 & $\mathrm{C}_{7} \mathrm{H}_{15} \mathrm{O}_{2}+\mathrm{O}_{2}=\mathrm{C}_{7} \mathrm{ket}_{12}+\mathrm{OH}$ & $4.50 \cdot 10^{14}$ & 0.0 & 18232.7 & 43 & $\mathrm{C}_{2} \mathrm{H}_{5}+\mathrm{O}_{2}=\mathrm{C}_{2} \mathrm{H}_{4}+\mathrm{HO}_{2}$ & $2.00 \cdot 10^{10}$ & 0.0 & -2200.0 \\
\hline 7 & $\mathrm{C}_{7} \mathrm{ket}_{12}=\mathrm{C}_{5} \mathrm{H}_{11} \mathrm{CO}+\mathrm{CH}_{2} \mathrm{O}+\mathrm{OH}$ & $9.53 \cdot 10^{14}$ & 0.0 & 41100.0 & 44 & $\mathrm{CH}_{4}+\mathrm{O}_{2}=\mathrm{CH}_{3}+\mathrm{HO}_{2}$ & $7.90 \cdot 10^{13}$ & 0.0 & 56000.0 \\
\hline 8 & $\mathrm{C}_{5} \mathrm{H}_{11} \mathrm{CO}=\mathrm{C}_{2} \mathrm{H}_{4}+\mathrm{C}_{3} \mathrm{H}_{7}+\mathrm{CO}$ & 9 & 0.0 & 00.0 & 45 & $\mathrm{OH}+\mathrm{HO}_{2}=\mathrm{H}_{2} \mathrm{O}+\mathrm{O}_{2}$ & 12 & 0.0 & 0.0 \\
\hline 9 & $\mathrm{C}_{7} \mathrm{H}_{15}-2=\mathrm{C}_{2} \mathrm{H}_{5}+\mathrm{C}_{2} \mathrm{H}_{4}+\mathrm{C}_{3} \mathrm{H}_{6}$ & $7.04 \cdot 10^{14}$ & 0.0 & 34600.0 & 46 & $\mathrm{CH}_{3}+\mathrm{O}_{2}=\mathrm{CH}_{2} \mathrm{O}+\mathrm{OH}$ & $3.80 \cdot 10^{11}$ & 0.0 & 9000.0 \\
\hline 10 & $\mathrm{C}_{3} \mathrm{H}_{7}=\mathrm{C}_{2} \mathrm{H}_{4}+\mathrm{CH}_{3}$ & 9.60 . & 0.0 & 30950.0 & 47 & $\mathrm{CH}_{4}+\mathrm{H}=\mathrm{CH}_{3}+\mathrm{H}_{2}$ & 6.60 & 1.6 & 10840.0 \\
\hline 11 & $\mathrm{C}_{3} \mathrm{H}_{7}=\mathrm{C}_{3} \mathrm{H}_{6}+\mathrm{H}$ & $1.25 \cdot 10^{14}$ & 0.0 & 36900.0 & 48 & $\mathrm{CH}_{4}+\mathrm{H}=\mathrm{CH}_{3}+\mathrm{H}_{2}$ & $1.60 \cdot 10^{6}$ & 2.1 & 2460.0 \\
\hline 12 & $\mathrm{C}_{3} \mathrm{H}_{6}+\mathrm{CH}_{3}=\mathrm{C}_{3} \mathrm{H}_{5}+\mathrm{CH}_{4}$ & $9.00 \cdot 10^{12}$ & 0.0 & 8480.0 & 49 & $\mathrm{CH}_{4}+\mathrm{O}=\mathrm{CH}_{3}+\mathrm{OH}$ & $1.02 \cdot 10^{9}$ & 1.5 & 8604.0 \\
\hline 13 & $\mathrm{C}_{3} \mathrm{H}_{5}+\mathrm{O}_{2}=\mathrm{C}_{3} \mathrm{H}_{4}+\mathrm{HO}_{2}$ & $6.00 \cdot 10^{11}$ & 0.0 & 0.0 & 50 & $\mathrm{CH}_{4}+\mathrm{HO}_{2}=\mathrm{CH}_{3}+\mathrm{H}_{2} \mathrm{O}_{2}$ & 11 & 0.0 & .0 \\
\hline 14 & $\mathrm{C}_{3} \mathrm{H}_{4}+\mathrm{OH}=\mathrm{C}_{2} \mathrm{H}_{3}+\mathrm{CH}_{2} \mathrm{O}$ & $1.00 \cdot 10^{12}$ & 0.0 & 0.0 & 51 & $\mathrm{CH}_{4}+\mathrm{CH}_{2}=\mathrm{CH}_{3}+\mathrm{CH}_{3}$ & $4.00 \cdot 10^{12}$ & 0.0 & -570.0 \\
\hline 15 & $\mathrm{C}_{3} \mathrm{H}_{4}+\mathrm{OH}=\mathrm{C}_{2} \mathrm{H}_{4}+\mathrm{HCO}$ & 1.00 & 0.0 & 0.0 & 52 & $\mathrm{C}_{3} \mathrm{H}_{6}=\mathrm{C}_{2} \mathrm{H}_{3}+\mathrm{CH}_{3}$ & 15 & 0.0 & 5500.0 \\
\hline 16 & $\mathrm{CH}_{3}+\mathrm{HO}_{2}=\mathrm{CH}_{3} \mathrm{O}+\mathrm{OH}$ & 5.00 & 0.0 & 0 & 3 & $\mathrm{CH}_{2}+$ & $3.15 \cdot 10^{15}$ & 0.0 & 300.0 \\
\hline 17 & $\mathrm{CH}_{3}+\mathrm{OH}=\mathrm{CH}_{2}+\mathrm{H}_{2} \mathrm{O}$ & $7.50 \cdot 10^{6}$ & 2.0 & 000.0 & 54 & $\mathrm{CH}_{2}+\mathrm{CH}_{2}=\mathrm{C}_{2} \mathrm{H}_{2}+\mathrm{H}+\mathrm{H}$ & $1.20 \cdot 10^{14}$ & 0.0 & 800.0 \\
\hline 18 & $\mathrm{CH}_{2}+\mathrm{OH}=\mathrm{CH}_{2} \mathrm{O}+\mathrm{H}$ & 2 & 0.0 & 0 & 5 & $\mathrm{C}_{2} \mathrm{H}_{4}+\mathrm{M}=\mathrm{C}_{2} \mathrm{H}_{2}+\mathrm{H}_{2}+\mathrm{M}$ & 15 & 0.0 & 00.0 \\
\hline 19 & $\mathrm{CH}_{2}+\mathrm{O}_{2}=\mathrm{HCO}+\mathrm{OH}$ & $4.30 \cdot 10^{10}$ & 0.0 & 00.0 & 56 & $\mathrm{C}_{2} \mathrm{H}_{2}+\mathrm{O}_{2}=\mathrm{HCO}+\mathrm{HCO}$ & $4.00 \cdot 10^{12}$ & 0.0 & 28000.0 \\
\hline 20 & $\mathrm{CH}_{2}+\mathrm{O}_{2}=\mathrm{CO}_{2}+\mathrm{H}_{2}$ & $6.90 \cdot 10^{11}$ & 0.0 & 500.0 & 57 & $\mathrm{C}_{2} \mathrm{H}_{2}+\mathrm{O}=\mathrm{CH}_{2}+\mathrm{CO}$ & $1.02 \cdot 10^{1}$ & 2.0 & 1900.0 \\
\hline 21 & $\mathrm{CH}_{2}+\mathrm{O}_{2}=\mathrm{CO}+\mathrm{H}_{2} \mathrm{O}$ & 0 & 0.0 & 0 & 58 & $\mathrm{C}_{2} \mathrm{H}_{2}+\mathrm{H}+\mathrm{M}=\mathrm{C}_{2} \mathrm{H}_{3}+\mathrm{M}$ & 5.5 & 0.0 & 2410.0 \\
\hline 22 & $\mathrm{CH}_{2}+\mathrm{O}_{2}=\mathrm{CH}_{2} \mathrm{O}+\mathrm{O}$ & $.00 \cdot 10^{13}$ & 0.0 & 000.0 & 59 & $\mathrm{C}_{2} \mathrm{H}_{3}+\mathrm{H}=\mathrm{C}_{2} \mathrm{H}_{2}+\mathrm{H}_{2}$ & $4.00 \cdot 10^{13}$ & 0.0 & 0.0 \\
\hline 23 & $\mathrm{CH}_{2}+\mathrm{O}_{2}=\mathrm{CO}_{2}+\mathrm{H}+\mathrm{H}$ & $5.00 \cdot 10^{13}$ & 0.0 & 1000.0 & 60 & $\mathrm{C}_{2} \mathrm{H}_{3}+\mathrm{OH}=\mathrm{C}_{2} \mathrm{H}_{2}+\mathrm{H}_{2} \mathrm{O}$ & $3.00 \cdot 10^{13}$ & 0.0 & 0.0 \\
\hline 24 & $\mathrm{CH}_{2}+\mathrm{O}_{2}=\mathrm{CO}+\mathrm{OH}+\mathrm{H}$ & 10 & 0.0 & 00.0 & 61 & $\mathrm{C}_{2} \mathrm{H}_{3}+\mathrm{CH}_{2}=\mathrm{C}_{2} \mathrm{H}_{2}+\mathrm{CH}_{3}$ & 013 & 0.0 & .0 \\
\hline 25 & $\mathrm{CH}_{3} \mathrm{O}+\mathrm{CO}=\mathrm{CH}_{3}+\mathrm{CO}_{2}$ & $1.57 \cdot 10^{14}$ & 0.0 & 11800.0 & 62 & $\mathrm{C}_{2} \mathrm{H}_{3}+\mathrm{C}_{2} \mathrm{H}_{3}=\mathrm{C}_{2} \mathrm{H}_{2}+\mathrm{C}_{2} \mathrm{H}_{4}$ & $1.45 \cdot 10^{13}$ & 0.0 & 0.0 \\
\hline 26 & $\mathrm{CO}+\mathrm{OH}=\mathrm{CO}_{2}+\mathrm{H}$ & $8.99 \cdot 10^{\prime}$ & 1.3 & -758.0 & 63 & $\mathrm{C}_{2} \mathrm{H}_{3}+\mathrm{O}=\mathrm{C}_{2} \mathrm{H}_{2}+\mathrm{OH}$ & $1.00 \cdot 10^{13}$ & 0.0 & 0.0 \\
\hline 27 & $\mathrm{O}+\mathrm{OH}=\mathrm{O}_{2}+\mathrm{H}$ & 14 & -0.5 & 0.0 & 64 & $\mathrm{C}_{2} \mathrm{H}_{2}+\mathrm{OH}=\mathrm{CH}_{3}+\mathrm{CO}$ & $0^{-4}$ & 4.0 & -2000.0 \\
\hline 28 & $\mathrm{H}+\mathrm{HO}_{2}=\mathrm{OH}+\mathrm{OH}$ & $1.70 \cdot 10^{14}$ & 0.0 & 875.0 & 65 & $\mathrm{C}_{2} \mathrm{H}_{3}=\mathrm{C}_{2} \mathrm{H}_{2}+\mathrm{H}$ & $4.60 \cdot 10^{40}$ & -8.8 & 46200.0 \\
\hline 29 & $\mathrm{OH}+\mathrm{OH}=\mathrm{O}+\mathrm{H}_{2} \mathrm{O}$ & $00 \cdot 10^{\circ}$ & 1.3 & 0.0 & 66 & $\mathrm{~N}+\mathrm{NO}<=>\mathrm{N}_{2}+\mathrm{O}$ & $0^{13}$ & 0.0 & 330.0 \\
\hline 30 & $\mathrm{H}+\mathrm{O}_{2}+\mathrm{M}=\mathrm{HO}_{2}+\mathrm{M}$ & $3.60 \cdot 10^{17}$ & -0.7 & 0.0 & 67 & $\mathrm{~N}+\mathrm{O}_{2}<=>\mathrm{NO}+\mathrm{O}$ & $2.65 \cdot 10^{13}$ & 0.0 & 6400.0 \\
\hline 31 & $\mathrm{H}_{2} \mathrm{O}_{2}+\mathrm{M}=\mathrm{OH}+\mathrm{OH}+\mathrm{M}$ & $1.00 \cdot 10^{16}$ & 0.0 & 45500.0 & 68 & $\mathrm{~N}_{2} \mathrm{O}+\mathrm{O}<=>2 \mathrm{NO}$ & $2.90 \cdot 10^{13}$ & 0.0 & 23150.0 \\
\hline 32 & $\mathrm{H}_{2}+\mathrm{OH}=\mathrm{H}_{2} \mathrm{O}+\mathrm{H}$ & $1.17 \cdot 10^{9}$ & 1.3 & 26.0 & 69 & $\mathrm{~N}_{2} \mathrm{O}+\mathrm{OH}<=>\mathrm{N}_{2}+\mathrm{HO}_{2}$ & $2.00 \cdot 10^{12}$ & 0.0 & 21060.0 \\
\hline 33 & $\mathrm{HO}_{2}+\mathrm{HO}_{2}=\mathrm{H}_{2} \mathrm{O}_{2}+\mathrm{O}_{2}$ & $3.00 \cdot 10^{12}$ & 0.0 & 0.0 & 70 & $\mathrm{~N}_{2} \mathrm{O}(+\mathrm{M})<=>\mathrm{N}_{2}+\mathrm{O}(+\mathrm{M})$ & $1.30 \cdot 10^{11}$ & 0.0 & 59620.0 \\
\hline 34 & $\mathrm{CH}_{2} \mathrm{O}+\mathrm{OH}=\mathrm{HCO}+\mathrm{H}_{2} \mathrm{O}$ & $5.56 \cdot 10^{10}$ & 1.1 & -76.5 & 71 & $\mathrm{HO}_{2}+\mathrm{NO}<\Rightarrow \mathrm{NO}_{2}+\mathrm{OH}$ & $2.11 \cdot 10^{12}$ & 0.0 & -480.0 \\
\hline 35 & $\mathrm{CH}_{2} \mathrm{O}+\mathrm{HO}_{2}=\mathrm{HCO}+\mathrm{H}_{2} \mathrm{O}_{2}$ & $3.00 \cdot 10^{12}$ & 0.0 & 0 & 72 & $\mathrm{NO}+\mathrm{O}+\mathrm{M}<=>\mathrm{NO}_{2}+\mathrm{M}$ & $1.06 \cdot 10^{20}$ & -1.4 & 0 \\
\hline 36 & $\mathrm{HCO}+\mathrm{O}_{2}=\mathrm{HO}_{2}+\mathrm{CO}$ & $3.30 \cdot 10^{13}$ & -0.4 & 0.0 & 73 & $\mathrm{NO}_{2}+\mathrm{O}<=>\mathrm{NO}+\mathrm{O}_{2}$ & $3.90 \cdot 10^{12}$ & 0.0 & -240.0 \\
\hline 37 & $\mathrm{HCO}+\mathrm{M}=\mathrm{H}+\mathrm{CO}+\mathrm{M}$ & $1.59 \cdot 10^{18}$ & 0.9 & 56712.3 & 74 & $\mathrm{NO}_{2}+\mathrm{H}<=>\mathrm{NO}+\mathrm{OH}$ & $1.32 \cdot 10^{14}$ & 0.0 & 360.0 \\
\hline
\end{tabular}


мировались по неявной схеме первого порядка точности. Диффузионные члены аппроксимировались по схеме второго порядка.

\section{2. Постановка и результаты моделирования}

Исследование проведено для широко применяемого в дизель-генераторных установках дизельного двигателя в широком диапазоне частоты вращения коленчатого вала. Был рассмотрен рядный шестицилиндровый дизельный двигатель. Рабочий объем всех цилиндров 6.65 л. Диаметр цилиндра 105 мм, ход поршня 128 мм, длина шатуна 320 мм. Степень сжатия 17.5. Частота вращения коленчатого вала при номинальной мощности 2300 об/мин. Форсунка закрытого типа с многоструйным распылением, количество струй 7, диаметр сопла 0.182 мм. Максимальное давление впрыска, обеспечиваемое топливной системой, 180 МПа. Температура окружающей среды в расчетах была равна $293 \mathrm{~K}$. Температура свежего заряда с учетом охлаждения воздуха в охладители принята равной $399 \mathrm{~K}$. Средняя температура стенок $450 \mathrm{~K}$.

Для моделирования процессов, происходящих в камере сгорания, была разработана математическая модель процессов горения в камере сгорания с учетом характеристик впрыска, воспламенения и сгорания топливной смеси. Построена пространственная геометрия расчетной области, представляющая внутреннюю часть камеры сгорания дизельного двигателя. Поскольку геометрия осесимметрична, с целью сокращения расчетного времени был взят сектор в $30^{\circ}$ (рис. 1 ). Для расчетов использована многоблочная структурированная криво-

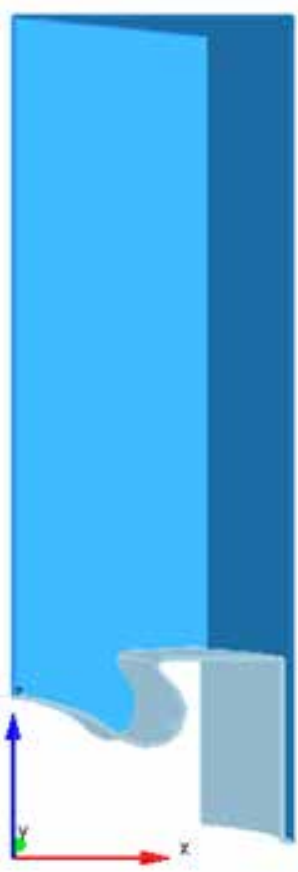

a)

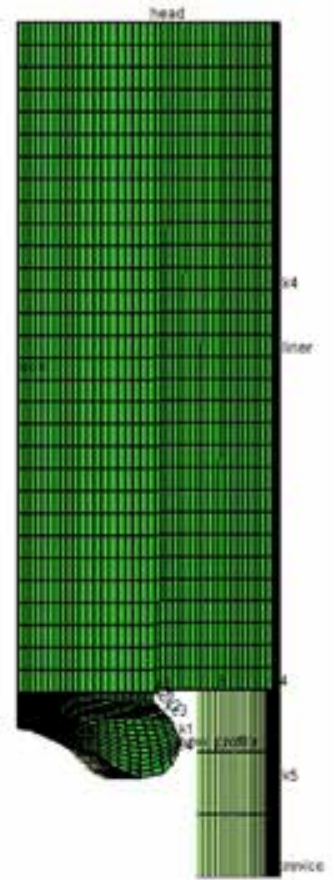

б)

Рис. 1. Геометрия расчетной области (а) и расчетная сетка (б)

Fig. 1. The geometry of the computational domain (a) and the computational grid (б) 
линейная сетка, состоящая из 200000 расчетных ячеек (рис. 1б). Методические исследования показали, что такой детализации вполне достаточно для получения решения, не зависящего от сетки.

Особенностью моделирования процессов в камере сгорания двигателя является необходимость учитывать изменение объема расчетной области в процессе движения поршня. В данной работе для расчета процесса движения поршня использовался лагранжев метод динамических сеток (ALE). В процессе движения поршня расчетные ячейки деформируются вместе с уменьшением объема камеры сгорания. Процесс сжатия и расширения газов описывается уравнением состояния для смеси идеальных газов. Задача существенно нестационарная. На рис. 2 в качестве примера показано поведение поля температуры в камере сгорания в процессе сжатия газа, впрыска и воспламенения топлива и расширения продуктов сгорания при частоте вращения коленчатого вала 1800 об/мин. Как видно, поле температуры после начала процесса горения топлива достаточно неоднородно.

Разработанная математическая модель процессов тепломассообмена в камере сгорания учитывает характеристики впрыска дизельного двигателя. На рис. 3 показаны результаты моделирования процесса впрыска, испарения и воспламенения дизельного топлива в процессе движения поршня. В результате моделирования можно описать поведение основных характеристик спрея: фракционный состав, установить фазы распыла и их длительности, угол и глубину проникновения распыла, заполненность факела спрея каплями топлива и т.д. Полученные данные позволяют проводить оптимизацию работы топливных форсунок и настраивать характеристики впрыска с целью получения наиболее эффективных параметров работы двигателя в различных режимах работы в составе генераторной установки. Определение количества впрыскиваемого топлива и момента впрыска чрезвычайно важно для управления работой дизельного двигателя. Ранний предварительный впрыск топлива положительно влияет на шумность работы двигателя, но приводит к увеличению выбросов сажи. При слишком позднем впрыске топлива существенно возрастает его недожег. В результате этого снижается КПД, увеличивается закоксованность двигателя и сокращается интервал замены масла.
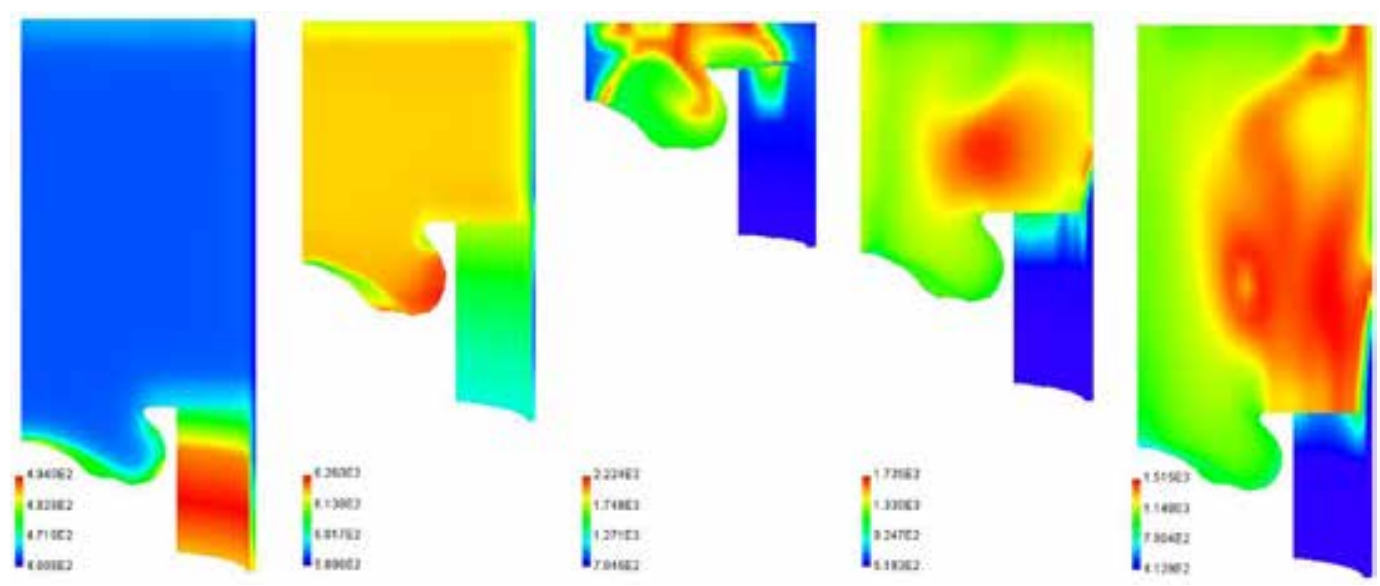

Рис. 2. Поле температуры в камере сгорания во время сжатия и рабочего хода поршня

Fig. 2. Temperature field in the combustion chamber during compression and piston stroke 

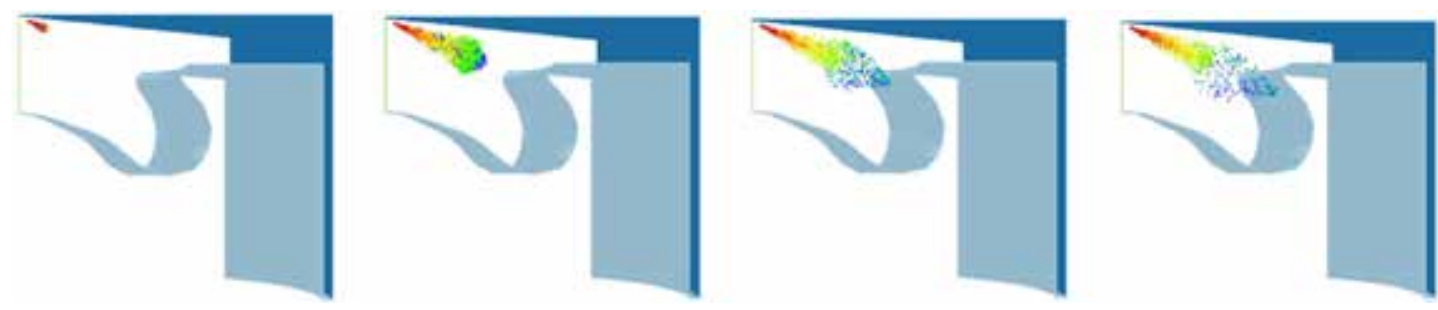

Рис. 3. Характеристики распыла дизельного топлива в камере сгорания

Fig. 3. Characteristics of diesel fuel spray in the combustion chamber

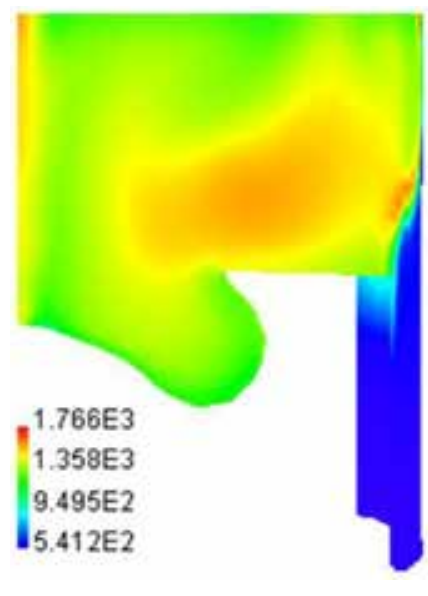

a

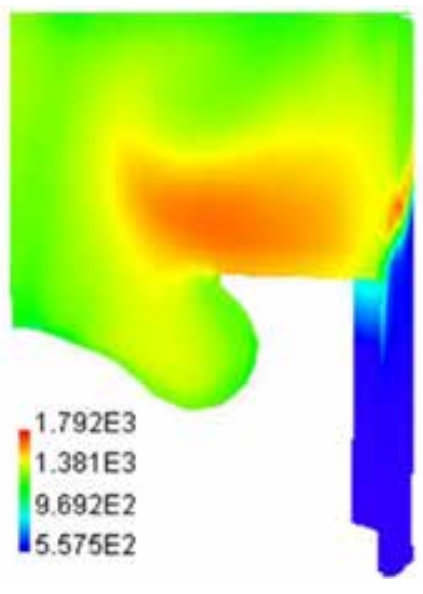

b

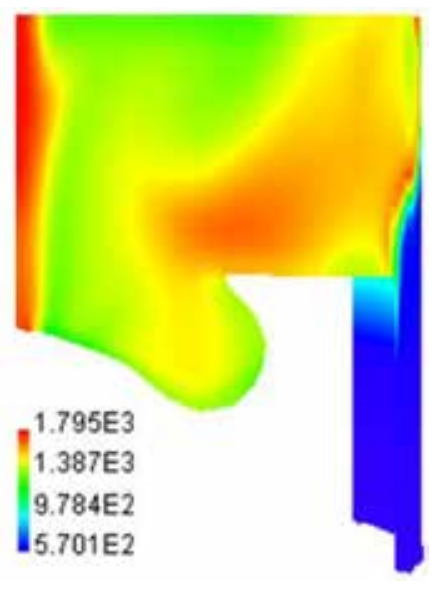

c

Рис. 4. Температура в камере сгорания дизель-генератора в зависимости от частоты вращения коленчатого вала (a - $1000 \mathrm{rpm}, \mathrm{b}-1800 \mathrm{rpm}, \mathrm{c}-2300 \mathrm{rpm})$

Fig. 4. Temperature in the combustion chamber of a diesel generator depending on the rotational speed of the crankshaft (a - $1000 \mathrm{rpm}, \mathrm{b}-1800 \mathrm{rpm}, \mathrm{c}-2300 \mathrm{rpm})$

Далее было проведено исследование локальных тепло-газодинамических характеристик двигателя в различных режимах его работы. На рис. 4-6 в качестве примера показаны локальные распределения температуры, массовой доли частиц сажи и окислов азота в камере сгорания для трех различных режимов работы двигателя. Видно, что от режима работы дизель-генератора значительно зависят его экологические характеристики. В режимах, далеких от оптимальных, существенно возрастают выбросы вредных веществ. Так, в частности, было показано, что локальные значения выбросов сажи при увеличении частоты вращения коленчатого вала с 1000 до 2300 об/мин снижаются более чем 2 раза, локальные значения окислов азота - в 1.7 раза, недожег высокомолекулярных углеводородов - на 30 \%. При этом разброс локальных значений температуры в камере сгорания не превышает 100 градусов.

Локальные характеристики не только неоднородно распределены по объему камеры сгорания, но и очевидно значительно меняются во времени в процессе сжатия, впрыска топлива и выпуска отработанных газов. Количественные данные о поведении осредненных по объему камеры сгорания температуры и концентрации выбросов в процессе движения коленчатого вала в диапазоне углов от -180 до $120^{\circ}$ приведены на рис. 7. Как видно, осредненные по объему 


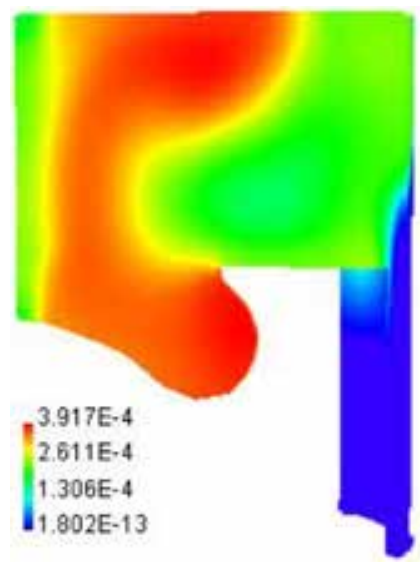

a

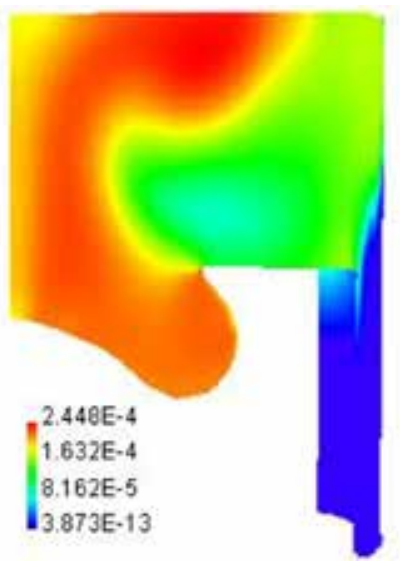

b

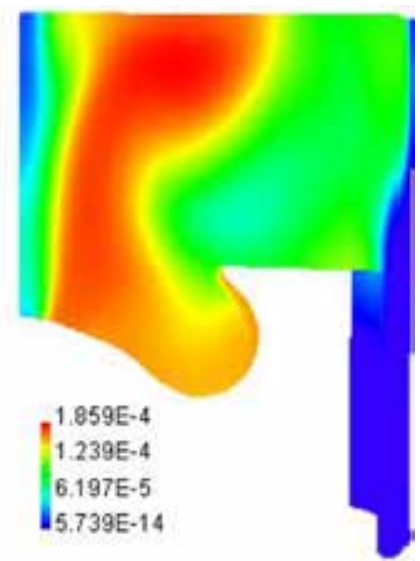

$\mathrm{c}$

Рис. 5. Массовая доля частиц сажи в камере сгорания дизель-генератора в зависимости от частоты вращения коленчатого вала (a - $1000 \mathrm{rpm}, \mathrm{b}-1800 \mathrm{rpm}, \mathrm{c}-2300 \mathrm{rpm})$

Fig. 5. Mass fraction of soot particles in the combustion chamber of a diesel generator depending on the rotational speed of the crankshaft ( $-1000 \mathrm{rpm}, \mathrm{b}-1800 \mathrm{rpm}, \mathrm{c}-2300 \mathrm{rpm})$

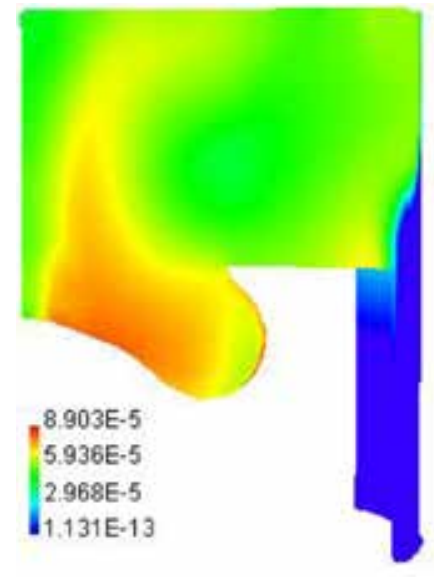

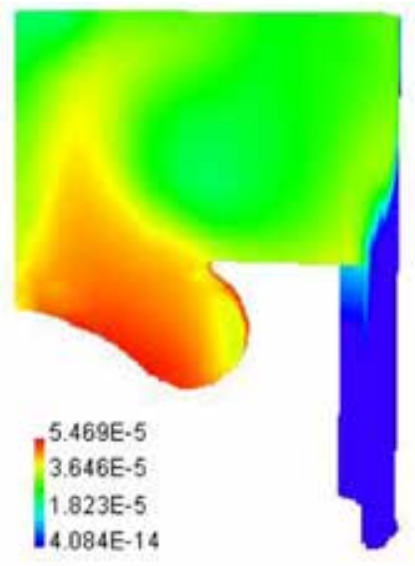

b

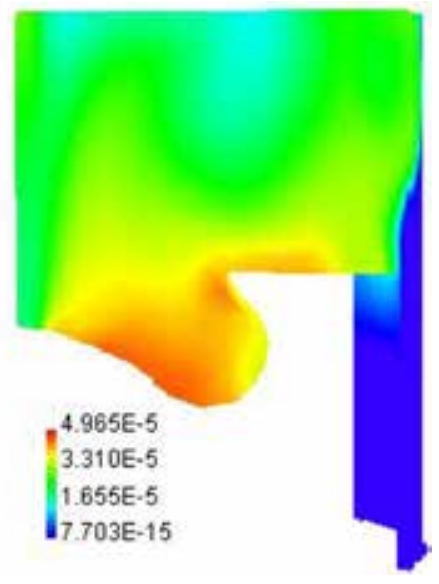

Рис. 6. Массовая доля окислов азота в камере сгорания дизель-генератора в зависимости от частоты вращения коленчатого вала (a - $1000 \mathrm{rpm}, \mathrm{b}-1800 \mathrm{rpm}, \mathrm{c}-2300 \mathrm{rpm})$

Fig. 6. Mass fraction of nitrogen oxides in the combustion chamber of a diesel generator depending on the rotational speed of the crankshaft (a-1000 rpm, b- $1800 \mathrm{rpm}, \mathrm{c}-2300 \mathrm{rpm})$

камеры сгорания характеристики также существенно зависят от режима работы двигателя. С увеличением частоты вращения коленчатого вала снижаются средние значения концентраций основных выбросов. При этом сам вид кривых в зависимости от положения поршня при разных значениях скорости вращения вала в целом схожий.

Далее было проведено исследование интегральных характеристик дизель-генератора в широком диапазоне режимов его работы. На рис. 8 приведены расчетные интегральные характеристики двигателя в зависимости от частоты оборотов коленчатого вала. Как видно, в диапазоне регулирования от 1000 до 2300 об/мин силовые и энергетические характеристики 


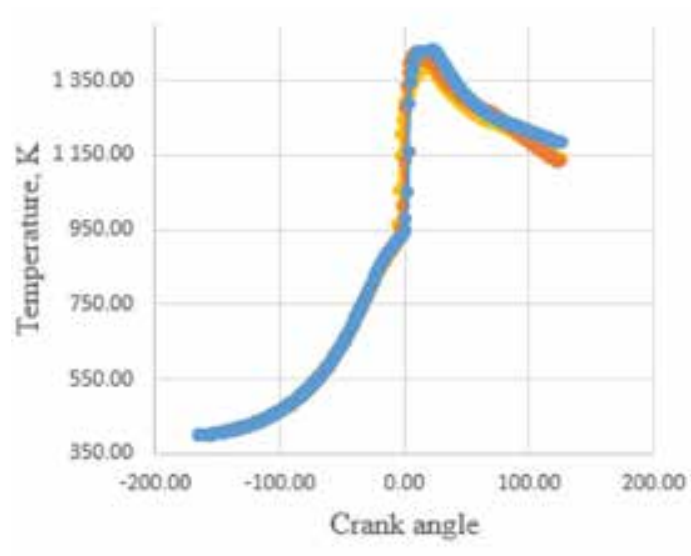

a)

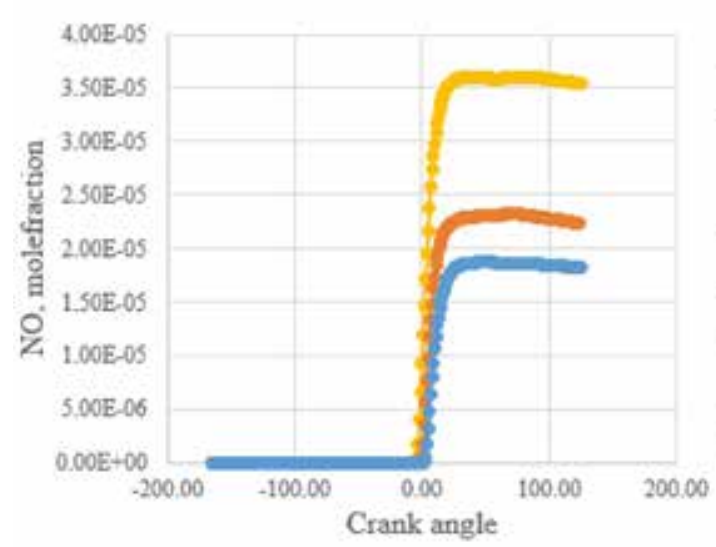

B)

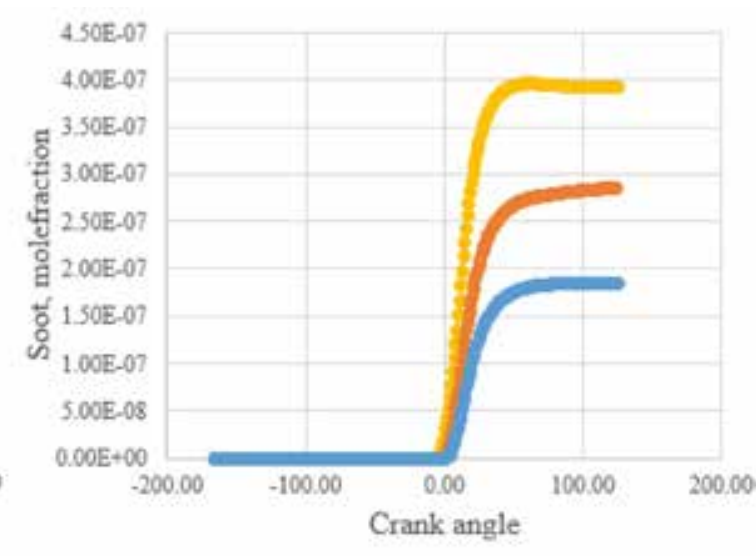

б)

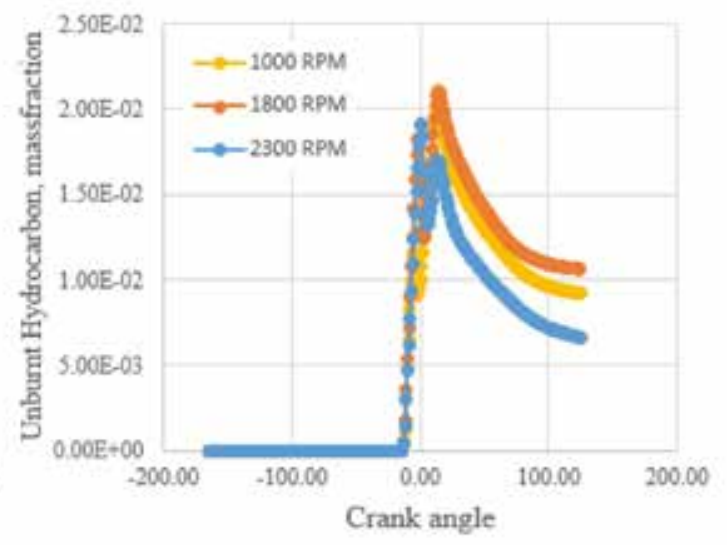

г)

Рис. 7. Средние значения температуры (а), объемной доли сажи (б), окислов азота (в) и недожега топлива (г) в камере сгорания в зависимости от угла поворота

Fig. 7. Average values of temperature (a), volume fraction of soot (б), nitrogen oxides (в) and underburnt hydrocarbon $(\Gamma)$ in the combustion chamber at different rotational speed of the crankshaft

меняются очень значительно. При таких условиях использование генераторных установок с переменной частотой вращения будет наиболее оптимальным, поскольку это позволяет максимально эффективно использовать дизельный двигатель, подстраивая его под электрическую нагрузку сети.

Однако при увеличении оборотов коленчатого вала возрастает и количество подаваемого камеру сгорания топлива и воздушной смеси. Поэтому несмотря на то, что локальные значения вредных выбросов в режимах с пониженной частотой вращения могут быть ниже, валовые выбросы с увеличением частоты вращения, как правило, возрастают. В связи с этим для оптимизации работы дизель-генераторных установок с точки зрения экологических характеристик более рационально анализировать приведенные к мощности значения выбросов. На рис. 9 отражена расчетная зависимость удельных выбросов сажи. Как видно, удельные выбросы в данном случае возрастают, в то время как выбросы окислов азота снижаются. Таким образом, при совершенствовании дизель-генераторов с переменной частотой вращения необходимо учитывать 


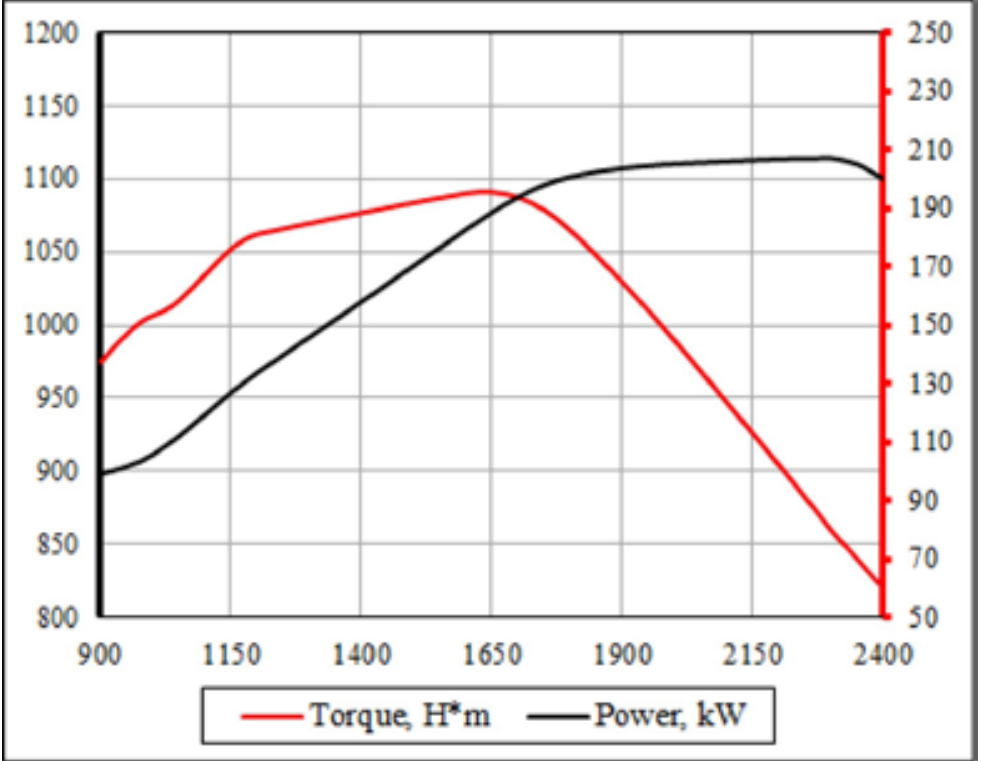

Рис. 8. Зависимость мощности и крутящего момента от оборотов коленчатого вала

Fig. 8. Dependence of power and torque on the crankshaft speed

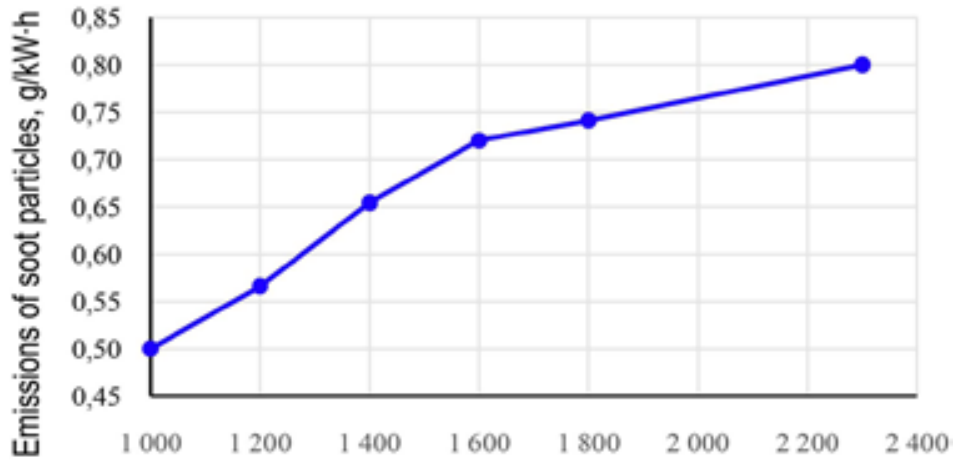

Rotational speed of rotation, rpm

Рис. 9. Зависимость приведенных к мощности установки выбросов частиц сажи от частоты вращения коленчатого вала

Fig. 9. Dependence of the soot particle emissions resulted in the power of diesel generator on the crankshaft speed

множество факторов, нелинейно связанных между собой, включая и экологические характеристики. Разработанная математическая модель позволяет проводить такого рода оптимизацию.

\section{Заключение}

Разработана математическая модель процессов тепломассообмена в камере сгорания дизель-генераторных установок с вентильно-индукторными генераторами. В отличие от известных ранее моделей, основанных на решении систем обыкновенных дифференциальных уравнений, данная математическая модель основана на методах вычислительной гидродинамики 
(CFD). Модель учитывает реальную геометрию камеры сгорания и условия работы дизельного двигателя, характеристики работы форсунок и свойства дизельного топлива. С помощью разработанной модели проведено исследование основных характеристик дизель-генератора в широком диапазоне режимов его работы. Частота вращения коленчатого вала в расчетах варьировалась от 900 до 2400 об/мин. Проанализированы характеристики распыла, дробления и воспламенения топлива в камере сгорания. Исследованы особенности поведения локальных и интегральных характеристик камеры сгорания двигателя. При этом рассмотрены не только его энергетические характеристики, но и экологические параметры. Данная работа является первым шагом к созданию полноценного цифрового двойника дизель-генераторной установки с переменной частотой вращения. В дальнейшем разработанная математическая модель двигателя будет объединена с математической моделью электрической части генератора и на этой основе будет разработан цифровой двойник энергетической установки с вентильно-индукторным генератором.

\section{Благодарности / Acknowledgements}

Данный проект поддержан за счет средств, предоставленных РФФИ, Правительством Красноярского края, Краевым фондом науки и ООО «Нижнебогучанская ГЭС» в рамках научного проекта № 20-48-242909 «Теоретические основы построения адаптивных регуляторов частоты вращения и качества генерируемой электроэнергии дизель-генераторных установок с вентильно-индукторными генераторами».

This project was supported by the Russian Foundation for Basic Research, the Government of the Krasnoyarsk Territory, the Regional Science Foundation and LLC «Nizhneboguchanskaya HPP» in the framework of the research project No. 20-48-242909 «Theoretical foundations for the construction of adaptive controllers of the speed and quality of generated electricity for diesel generator units with valve-inductor generators».

\section{Список литературы / References}

[1] Орлов А.В., Путятинский В.А., Сапожников В.В. Перспективы создания дизельэлектрических установок с переменной частотой вращения, Судостроение, 1976, 10, 28-29 [Orlov A.V., Putyatinskiy V.A., Sapozhnikov V.V. Prospects for the creation of variable speed diesel electric installations, Shipbuilding, 1976, 10, 28-29 (in Russian)]

[2] Крутов В.И. Автоматическое регулирование двигателей внутреннего сгорания: учеб. пособие для втузов, М.: Машиностроение, 1979. 615 c. [Krutov V.I. Automatic regulation of internal combustion engines: a manual for technical colleges, Moscow, Mashinostroyeniye, 1979, 615 p. (in Russian)]

[3] Хватов О.С. и др. Высокоэффективная дизель-генераторная электростанция переменной частоты вращения на основе машины двойного питания, Приводная техника, 2010, 5, 14-19. [Khvatov O.S. et al. Highly efficient diesel-generator power plant of variable speed based on a dual-feed machine, Drive technology, 2010, 5, 14-19 (in Russian)]

[4] Хватов О.С., Дарьенков А.Б. Интеллектуальные средства управления высокоэффективной дизельгенераторной установкой переменной частоты вращения, Известия ТулГУ. Сер. Технические науки. Bып. 3: в 5 ч, Тула: Изд-во ТулГУ, 2010, Ч. 4. С. 126-131. [Khvatov O.S., 
Darjenkov A.B. Intelligent controls for a highly efficient variable speed diesel generator unit, Izvestiya of TulSU. Ser. Technical sciences. Issue 3: in 5 p., Tula: Publishing house of TulSU, 2010, Part 4. P. 126-131 (in Russian)]

[5] Марков В.А., Девянин С.Н., Михальский Л.Л. Анализ комплексной системы автоматического регулирования частоты вращения вала дизельного двигателя и температуры охлаждающей жидкости, Инженерный журнал: наука и инноващии, 2013, 5, 1-19. URL: http://engjournal. ru/catalog/machin/criogen/724.html [Markov V.A., Devyanin S.N., Mikhalskiy L.L. Analysis of a complex system for automatic control of the shaft rotation speed of a diesel engine and the temperature of the coolant, Engineering Journal: Science and Innovations, 2013, 5, 1-19. URL: http://engjournal. $\mathrm{ru} / \mathrm{catalog} / \mathrm{machin} / \mathrm{criogen} / 724 . \mathrm{html}$ (in Russian)]

[6] Хватов О.С., Дарьенков А.Б., Тарпанов И.А., Пшеничников В.В. Имитационная модель дизель-генераторной электростанции переменной скорости вращения с интеллектуальной системой управления топливоподачей, Труды Нижегородского государственного технического университета им. Р.Е. Алексеева, 2011, 1(86), 185-192. [Khvatov O.S., Darjenkov A.B., Tarpanov I.A., Pshenichnikov V.V. A simulation model of a variable speed diesel generator power plant with an intelligent fuel supply control system, Proceedings of Nizhny Novgorod State Technical University named after R. E. Alekseev, 2011, 1 (86), 185-192 (in Russian)]

[7] Поляков И.С. Дизель-генераторная установка переменной частоты вращения. Дис. ... канд. техн. наук. Нижний Новгород, 2013. 155 с. [Polyakov I.S. Diesel generator unit of variable rotation speed. Dis. ... cand. tech. sciences. Nizhny Novgorod, 2013. 155 p. (in Russian)]

[8] Han Z., Reitz R.D. Turbulence modeling of internal combustion engines using RNG $\kappa-\varepsilon$ models. Combust. Sci. Technol., 1995, 106(4-6), 267-295.

[9] Sun Y., Reitz R.D. Modeling Low-Pressure Injections in Diesel HCCI Engines, ILASS Americas, 20th Annual Conference on Liquid Atomization and Spray Systems, Chicago, 2007.

[10]Ra Y., Reitz R. D.A vaporization model for discrete multi-component fuel sprays, Int. J. Multiphase Flow, 2009, 35, 101-117.

[11] Kong S.-C., Reitz R.D. Use of Detailed Chemical Kinetics to Study HCCI Engine Combustion With Consideration of Turbulent Mixing Effects, Trans. Am. Soc. Mech. Eng., 2002, 124, 702-707.

[12]Hiroyasu H. and Kadota T. Models for Combustion and Formation of Nitric Oxide and Soot in DI Diesel Engines, SAE Technical Paper 760129, SAE Technical Paper Series, 1976.

[13] Nagle J. Strickland-Constable R.F. Oxidation of Carbon Between 1000-2000 ${ }^{\circ}$, Proceedings of the Fifth Carbon Conference, Vol. 1, New York: Pergamon, 1962, pp. 154-164. 